\title{
Hospital Clostridium difficile infection (CDI) incidence as a risk factor for hospital-associated CDI
}

\author{
Aaron C. Miller, $\mathrm{PhD}^{\mathrm{a}}$, Linnea A. Polgreen, $\mathrm{PhD}^{\mathrm{b}}$, Joseph E. Cavanaugh, $\mathbf{P h D}^{\mathrm{c}}$, and $\mathrm{Philip}_{\mathrm{M}}$. \\ Polgreen, MD, MPH ${ }^{\mathrm{d},}{ }^{*}$ \\ aDepartment of Economics and Business, Cornell College, Mount Vernon, IA \\ bDepartment of Pharmacy Practice and Science, University of lowa, lowa City, IA \\ 'Department of Biostatistics, University of lowa, lowa City, IA \\ dDepartments of Internal Medicine and Epidemiology, University of lowa, lowa City, IA
}

\section{Abstract}

Background-Environmental risk factors for Clostridium difficile infections (CDIs) have been described at the room or unit level but not the hospital level. To understand the environmental risk factors for CDI, we investigated the association between institutional- and individual-level CDI.

Methods-We performed a retrospective cohort study using the Healthcare Cost and Utilization Project state inpatient databases for California (2005-2011). For each patient's hospital stay, we calculated the hospital CDI incidence rate corresponding to the patient's quarter of discharge, while excluding each patient's own CDI status. Adjusting for patient and hospital characteristics, we ran a pooled logistic regression to determine individual CDI risk attributable to the hospital's CDI rate.

Results-There were 10,329,988 patients (26,086 cases and 10,303,902 noncases) who were analyzed. We found that a percentage point increase in the CDI incidence rate a patient encountered increased the odds of CDI by a factor of 1.182 .

Conclusions-As a point of comparison, a 1-percentage point increase in the CDI incidence rate that the patient encountered had roughly the same impact on their odds of acquiring CDI as a 55.8day increase in their length of stay or a 60 -year increase in age. Patients treated in hospitals with a higher CDI rate are more likely to acquire CDI.

\section{Keywords}

Clostridium difficile infection; risk; health care-associated infection; environment

Clostridium difficile infections (CDIs) are a major cause of morbidity. ${ }^{1,2}$ Over the last decade, reports suggest that the disease is more common and perhaps more severe. ${ }^{3-9}$ The

*Address correspondence to Philip M. Polgreen, MD, MPH, Departments of Medicine and Epidemiology, University of Iowa, 200 Hawkins Dr, Iowa City, IA 52242. Philip-polgreen@uiowa.edu (P. Polgreen).

Conflicts of interest: None to report. 
disease is associated with a substantial cost, ${ }^{10-14}$ and CDI is now one of the most common health care-associated infections in the United States. ${ }^{2}$

Historically, the health care environment has been thought to contribute to the spread of CDI. Clostridium difficile spores can persist in health care environments for long periods of time ${ }^{15-17}$ and are resistant to most disinfectants and cleaning agents. ${ }^{18}$ Also, longer lengths of hospital stays have been associated with $\mathrm{CDI},{ }^{19-21}$ and occupying a room previously occupied by a CDI patient has been associated with CDI. ${ }^{22}$ Finally, CDI pressure, where pressure is characterized by a measure of the prevalence of CDI at the unit level, has been described as a risk factor for CDI. ${ }^{23,24}$ However, recent work using genome sequencing suggests that CDI symptomatic cases occurring within hospital settings are frequently not related, at least during times of endemic, nonoutbreak spread. ${ }^{25}$

Most investigations of environmental risk factors as a cause of CDI were performed at single centers and focus on individual rooms or hospital units rather than the whole hospital environment. However, patients frequently travel throughout hospitals, as do the health care workers caring for them. ${ }^{26-28}$ Therefore, unit-level measures of CDI incidence may fail to capture institution-wide risk and fail to address the possibility of an institutional-level environmental risk factor for CDI. Furthermore, the historical definition of a health care environment has been primarily focused on the physical environment: the presence or burden of spores. Perhaps a new, more expansive definition of the environment is needed: a definition that includes not only the physical environment but also the way health care is delivered within the unit or hospital.

Therefore, to gain a better understanding of the environment as a risk factor for CDI, we specifically investigated the association between the institutional incidence of CDI and CDI at an individual level. In other words, are patients hospitalized at institutions with a greater CDI incidence more likely to acquire CDI?

\section{METHODS}

This was a retrospective cohort study conducted using the Healthcare Cost and Utilization Project state inpatient database (SID) for the state of California from 2005-2011. Given the lack of personal identifiers in these data, the University of Iowa's Institutional Review Board considers this to be nonhuman subjects research. The SID contains records of inpatient discharges for community hospitals in California and covers all patients discharged between 2005 and 2011, regardless of payer type. Across the 7-year study window, these data comprise $>27.9$ million records. These records cover $>9.6$ million unique patients and 480 unique hospitals. Included are measures of a patient's principal and secondary diagnoses and procedures, demographics, length of stay (LOS), admission and discharge status, along with hospital charges and payment sources. The SID for California also features indicators on whether or not diagnoses contained in a patient record were present on admission (POA). Additionally, the California SID includes linking variables that allow multiple records of a given patient to be collected across time. Also, the set of all patient stays that occur in a specific hospital in a given month can be linked. 
This study was restricted to adult patients age $>18$ years. CDI cases were identified by the presence of a CDI diagnosis, indicated by the ICD-9-CM code 008.45. Because this study is concerned with hospital-acquired CDI, POA indicators were used to exclude cases that were potentially community-acquired CDI. Only CDI observations indicated as not POA were considered as cases. Additionally, to eliminate possible confounding effects associated with recurrent CDI, only the first recorded case of CDI for each patient was included. Therefore, once a patient was identified as a CDI case, all subsequent observations from that patient were excluded. CDI noncases were defined as those records without the presence of any CDI diagnosis. Patient records were pooled across time; therefore, an individual patient could contribute a single case observation and multiple noncase observations prior to receiving an initial CDI diagnosis. All records that contained a missing visit-linking variable were excluded as either a case or noncase observation because these patients could not be followed across the studied period.

All occurrences of CDI, both primary and secondary, and regardless of POA status or recurrence, were used when calculating hospital incidence rates. Each of these occurrences of CDI represents a potential for spread within the hospital. For each patient's hospital stay, we calculated the hospital CDI incidence rate corresponding to the year and quarter the patient was discharged. Hospital incidence rates were calculated as a quarterly average for each discharge quarter. Importantly, each patient's own CDI status was excluded from this rate to eliminate any positive bias when estimating the effect that this rate had on the likelihood of acquiring a CDI in small hospital settings.

Data were collected on a range of variables that have been previously analyzed as risk factors associated with CDI. Patient demographics that were assessed include age, sex, and nonwhite race. Characteristics of a patient's stay included LOS and a previous admission in the last 60 days. Procedures associated with CDI that were measured included dialysis, enteral feeding, intraabdominal or gastrointestinal procedures, mechanical ventilation, nasogastric tube insertion, and cancer chemotherapy. Finally, 2 sets of variables were used to measure a patient's comorbidities and severity of illness. First, we used the 30 Elixhauser comorbidity indicators created using ICD-9-CM codes that were developed for use with large administrative databases. ${ }^{29}$ The 30 comorbidities were used as separate indicator variables in the multivariable analysis. Second, we use the total number of procedures (NPR) and total number of diagnoses (NDX), excluding a CDI diagnosis, on a patient's record as a proxy measure for the patient's disease severity. We hypothesized that patients with more severe disease states will have a greater NDX and undergo a greater NPR during a stay. Because CDI patients would have one more recorded diagnosis, namely CDI, we excluded CDI diagnoses from the calculation of NPR. A thorough examination of the role of NDX and NPR in our analyses is given in Appendix 1.

In addition to patient-level characteristics, a number of hospital-specific characteristics were imputed and analyzed as possible risk factors for CDI as well. Hospital size was computed by each hospitals' total number of discharges in a given quarter. Hospital size was then broken into quintiles for analysis. Average patient age along with the average number of elderly and Medicaid patients were also calculated corresponding to each discharge quarter. 
We first conducted a univariable analysis to compare differences between CDI case patients and noncase patients without CDI. The significance differences between case and noncase patients for each risk factor was assessed using a $\chi^{2}$ test, for categorical variables, and the Mann-Whitney $U$ test, for quantitative variables. To analyze the effect of hospital CDI incidence on risk of CDI, while controlling for the effects of these risk factors, a multivariable analysis was conducted using logistic regression. Because patients could have multiple admissions over the 6-year study interval, the analysis was conducted as a pooled logistic regression, in a manner similar to the Framingham study cohort. ${ }^{30}$ In this setup, multiple admissions from a single patient that occurred prior to, and including, a first CDI diagnosis were treated as independent observations. This method has been previously used to analyze the risk factors associated with CDI. ${ }^{31}$ All statistical analyses were carried out using Stata SE version 13.1 (StataCorp, College Station, TX).

We conducted 2 sensitivity analyses. The first was to confirm that our results were not being driven by the omission of hospital-acquired CDI cases that were assigned as POA. To do this, we re-estimated our multivariable model using secondary cases of CDI, rather than using only those coded as not POA. Second, in our original analysis, we dropped a substantial number of observations without a hospital visit within 60 days ( $>8$ million). Therefore, for our second sensitivity analysis, we re-estimated our models including observations without previous visits.

\section{RESULTS}

The initial study population consisted of a total of 22,312,704 adult patients. Of these observations, 19,886,755 met all inclusion criteria, with 35,170 case observations identified as having a CDI marked as not POA and 19,851,585 noncase observations having no current, or previous, indication of CDI. A total of 10,329,988 of the observations (26,086 cases and 10,303,902 noncases) did not have missing values for any of the risk factors that were analyzed. There were $8,111,828$ observations excluded because they had no previous visit. These observations constituted the final study population that was used in the multivariable analysis. Table 1 provides a summary of the hospital CDI incidence rates (per 100 patients). In this table, CDI incidence is summarized across all patients with CDI coded not POA, all patients without any CDI, all patients (with or without any CDI), and hospital discharge quarters. The incidence rate encountered by cases was significantly greater $(P<$. 0001) than the rate encountered by noncases. The mean and median CDI incidence rates that was encountered by cases were $1.69 \%$ and $1.04 \%$, respectively, whereas the rates encountered by noncases were $0.89 \%$ and $0.79 \%$, respectively. Similarly, the first and third quartile incidence rates were greater for CDI cases than for the noncases. CDI incidence rates across hospital discharge quarters ranged from $0 \%-31.13 \%$, with mean and median rates of $1.06 \%$ and $0.59 \%$, respectively.

Nearly all of the variables analyzed were significantly different between cases and noncases in this univariable analysis. The CDI incidence that patients encountered was significantly greater among patients with CDI. Previously suggested risk factors for CDI, including LOS, previous admission within 60 days, intra-abdominal or gastrointestinal procedures, mechanical ventilation, nasogastric tube insertion, dialysis, cancer chemotherapy, along with 
enteral and parenteral nutrition, were all greater or more common among patients with CDI. Similarly, the nonroutine admission sources, from another hospital, a skilled nursing or immediate care, or emergency department, each occurred more frequently among CDI case patients. As hypothesized, both the mean NPR and NDX were greater among patients with CDI, and all but one of the comorbidity indicators (ie, drug abuse) were more common among case patients. Female sex and nonwhite race were less common among patients with CDI. Relatively more CDI cases occurred at small ( $<299$ discharges per quarter) and large $(>4,166$ discharges per quarter) hospitals. CDI cases occurred more frequently at hospitals with older patient populations, higher percentages of elderly patients, and lower percentages of Medicaid patients.

The variables that were significantly associated with CDI $(a<.05)$ in the final multivariable model are presented in Table 2. Consistent with the univariable findings, LOS, previous admission within 60 days, NPR, and NDX were associated with a significant increase in the odds of CDI, whereas nonwhite race was associated with decreased odds of CDI. In the multivariable results, female patients had increased odds of CDI. Adults $<45$ years of age had the lowest odds of CDI, and the odds of CDI increased with each consecutive age group $>45$ years old. Admission from another hospital or from skilled nursing or intermediate care were both associated with increased odds of CDI. Procedures that remained associated with increased odds of CDI included intra-abdominal or gastrointestinal procedure, mechanical ventilation, dialysis, cancer chemotherapy, and enteral or parenteral nutrition. In the univariable comparison, 27 out of 30 comorbidity indicators were significantly more common among patients with CDI. However, in the multivariable analysis, only 8 of the comorbidity indicators remained associated with increased odds of CDI, 13 were associated with decreased odds, and 9 were not significant. Further discussion of these variables is given in Appendix 1. Patients had increased odds of CDI as hospital size and hospital average patient age increased and had decreased odds of CDI at hospitals that treated a greater percentage of elderly patients and patients with Medicaid.

As suggested by the univariable analysis, the CDI incidence that a patient encountered in the hospital continued to be strongly associated with a significant increase in his or her odds for CDI when we controlled for other factors. The results of the multivariable analysis found that a percentage point increase in the CDI incidence rate a patient encountered increased his or her odds of CDI by a multiplicative factor of 1.182 . As a striking basis for comparison, a one percentage point increase in the CDI incidence rate that a patient encountered had roughly the same impact on their odds of acquiring a CDI as a 55.8-day increase in their LOS or a 60-year increase in age.

For our first sensitivity analysis, we examined the influence of POA indicators on our results. There were 35,170 CDI cases identified as having CDI not POA. We identified 151,244 total cases of CDI that also met our other inclusion criteria, 112,127 of which had CDI assigned as a secondary diagnosis. Therefore, the number of CDI cases identified as not POA appears markedly low, given previous findings on the prevalence of hospital-acquired CDI. A total of $85,968 \mathrm{CDI}$ cases coded as secondary or not POA met all inclusion criteria and did not have missing values for any variables. When these secondary CDI cases were included in the model, CDI incidence remained associated with a significant increase in the 
odds of CDI and had a similar effect size, with an odds ratio of 1.156. Although the effect of CDI incidence was slightly diminished, this is likely because of secondary CDI status capturing some CDI cases that were community associated.

Finally, our second sensitivity analysis including a larger data set (18,441,816 observations) produced results that were nearly identical to the original results. For example, the estimated odds of acquiring CDI were 1.186 for each percentage point increase in CDI incidence that the patient encountered.

\section{CONCLUSIONS}

Our results demonstrate that the odds of acquiring CDI during a hospital stay are strongly associated with the rate of CDI at that particular hospital. This risk remains significant even after adjusting for a wide range of both patient and hospital characteristics. Our model suggests that a mere one percentage point increase in a hospital's CDI rate significantly increases an individual's odds of acquiring CDI. The clinical significance of our result can be interpreted by comparing this result to the effects of more traditional CDI risk factors. For example, a one percentage point increase in the institution's CDI rate increases a patient's odds of acquiring CDI as much as an increase of 6 decades of age or adding $>50$ days to the patient's LOS. Finally a one percentage point increase in a hospital's CDI rate was associated with more risk than most of the comorbidities we considered, with all but a few exceptions, and the risk from the institution's rate remained significant controlling for other institutional risk factors known to be associated with CDI (eg, transfer from a skilled nursing facility).

A patient's environment has long been associated with risk of CDI. Spores can exist for long periods of time on inanimate objects and hospital surfaces. ${ }^{15,16}$ During CDI outbreaks, gowning and gloving of health care workers has helped prevent transmission. ${ }^{32}$ Also, epidemiologic studies within health care settings have implicated the environment for CDI spread, with roommates of CDI patients or patients occupying a room after a CDI patient being more likely to acquire the disease, ${ }^{22,33}$ and patients on units with more CDI being at greater risk. ${ }^{31}$ However, a study in a large health care system showed that most CDI cases were not genetically related, at least not the symptomatic cases of CDI, ${ }^{25}$ indicating that patient-to-patient spread may not be as common as previously thought.

Our results, however, suggest that the environment plays a role as a risk factor for CDI. However, perhaps, the definition of the health care environment needs to be expanded. The health care environment represents more than patients in close proximity. Patients closely related in a hospital setting are more likely to be treated by the same physicians with the same practice patterns and styles. Given that antibiotic use is a major risk factor for CDI, it is conceivable that some of the environmental risk may be secondary to local antibiotic prescribing patterns. For example, the risk for CDI is associated with the quantity of antibiotics used on a particular unit. ${ }^{34}$ Unfortunately, we are unable to control for hospitallevel antibiotic use directly because the family of Healthcare Cost and Utilization Project SIDs does not contain antibiotic prescribing. Therefore, we cannot rule out that a hospital's 
CDI rate is a surrogate marker for patterns of excessive antibiotic use. Indeed, we think this is probably likely and epidemiologically plausible.

There are several limitations associated with our study. First, we used administrative data to measure CDI. We do not know what medications these patients were taking, and we do not have microbiologic test results. We also do not know what type of CDI test was used, and rates of CDI depend on diagnostic tests that have changed over time. Also, with administrative data, it may be more difficult to ascertain whether cases of CDI were POA. Indeed, some cases of CDI appear to be acquired without any contact with the health care system. ${ }^{35,36}$ Second, we may be missing some CDI cases that are attributable to the hospital. ${ }^{37}$ Third, we only considered the first recorded case of CDI for each patient. This represents a conservative estimate. Fourth, we only used data from California, and these results may not be generalizable to other areas. Finally, a limitation associated with all such modeling efforts is that the estimates we generate for individual CDI risk associated with hospital CDI rates should be interpreted with some caution: additional measures may be necessary to accurately estimate the effect size. Future work should consider a mixed effects model using hospital as a random effect.

Despite our limitations, we demonstrate that patients treated in hospitals with a higher rate of CDI are more likely to acquire CDI, even after controlling for a broad range of individual and institutional factors. An institution's CDI rate is easy to measure, easy to observe, and based on our analysis, an institution's CDI rate has implications for patients at an individual level.

\section{Acknowledgments}

Funding/support: Supported by The National Heart, Lung and Blood Institute at the National Institutes of Health (K25 HL122305).

\section{APPENDIX 1 COMORBIDITY CAPTURED BY PROCEDURE AND DIAGNOSIS COUNTS}

Comorbidity is one of the most commonly cited risk factors for Clostridium difficile infection (CDI), and specific comorbidity indicators have previously been used as measures of comorbidity. In our univariable results, all but one of the comorbidities (drug abuse) was associated with an increased risk of CDI. This is expected in that comorbidities should increase the risk of hospital-acquired disease. In a multivariable model, 22 of the comorbidities were associated with a significant increased risk of CDI, whereas only drug abuse was associated with a significant decrease in risk. However, when the model included the total number of procedures (NPR) and total number of diagnoses (NDX), 13 of the comorbidity indicators were associated with a significant decreased risk of CDI, whereas only 8 of the comorbidity indicators were associated with an increased risk. This suggests that NPR and NDX, when included in the multivariable analysis, also capture some of the CDI risk associated with comorbidity. However, when NPR and NDX were not included in the model, the quality of fit for the model diminished substantially. Moreover, NPR and NDX were 2 of the most highly significant predictors of CDI in the multivariable model, in 
terms of both statistical significance (eg, $P$ value) and effect size. Because our primary objective was to evaluate CDI incidence as a risk factor, we opted to include both NPR and NDX in the final specification.

These findings, when NPR and NDX were added and removed from the multivariable model, are suggestive of 2 points. First, as hypothesized, the variables for NPR and NDX appear to capture some of the CDI risk associated with disease severity and comorbidity. Not only were NPR and NDX highly significant, they appear to better capture some of the CDI risk than many of the individual comorbidity indicators themselves. Second, many of the specific comorbidities that may, at first glance, appear to be risk factors for CDI appear to be protective when controlling for disease severity captured by NPR and NDX. Therefore, failing to include these, or similar measures of severity, may lead to an inappropriate interpretation of risk factors for CDI.

\section{References}

1. Elixhauser A, Jhung M. Clostridium difficile-associated disease in US hospitals, 1993-2005. 2008

2. Magill SS, Edwards JR, Bamberg W, Beldavs ZG, Dumyati G, Kainer MA, et al. Multistate pointprevalence survey of health care-associated infections. N Engl J Med. 2014; 370:1198-1208. [PubMed: 24670166]

3. Bartlett JG. Narrative review: the new epidemic of Clostridium difficile-associated enteric disease. Ann Intern Med. 2006; 145:758-764. [PubMed: 17116920]

4. McDonald LC, Killgore GE, Thompson A, Owens RC Jr, Kazakova SV, Sambol SP, et al. An epidemic, toxin gene-variant strain of Clostridium difficile. N Engl J Med. 2005; 353:2433-2441. [PubMed: 16322603]

5. Muto CA, Pokrywka M, Shutt K, Mendelsohn AB, Nouri K, Posey K, et al. A large outbreak of Clostridium difficile-associated disease with an unexpected proportion of deaths and colectomies at a teaching hospital following increased fluoroquinolone use. Infect Control Hosp Epidemiol. 2005; 26:273-280. [PubMed: 15796280]

6. Kuntz JL, Yang M, Cavanaugh J, Saftlas AF, Polgreen PM. Trends in Clostridium difficile infection among peripartum women. Infect Control Hosp Epidemiol. 2010; 31:532-534. [PubMed: 20350150]

7. McDonald LC, Owings M, Jernigan DB. Clostridium difficile infection in patients discharged from US short-stay hospitals, 1996-2003. Emerg Infect Dis. 2006; 12:409. [PubMed: 16704777]

8. Pepin J, Valiquette L, Alary ME, et al. Clostridium difficile-associated diarrhea in a region of Quebec from 1991 to 2003: a changing pattern of disease severity. CMAJ. 2004; 171:466-472. [PubMed: 15337727]

9. Ricciardi R, Rothenberger DA, Madoff RD, Baxter NN. Increasing prevalence and severity of Clostridium difficile colitis in hospitalized patients in the United States. Arc Surg. 2007; 142:624631.

10. Kyne L, Hamel MB, Polavaram R, Kelly CP. Health care costs and mortality associated with nosocomial diarrhea due to Clostridium difficile. Clin Infect Dis. 2002; 34:346-353. [PubMed: 11774082]

11. Song X, Bartlett JG, Speck K, Naegeli A, Carroll K, Perl TM. Rising economic impact of clostridium difficile-associated disease in adult hospitalized patient population. Infect Control Hosp Epidemiol. 2008; 29:823-828. [PubMed: 18643746]

12. Gabriel L, Beriot-Mathiot A. Hospitalization stay and costs attributable to Clostridium difficile infection: a critical review. J Hosp Infect. 2014; 88:12-21. [PubMed: 24996516]

13. Zimlichman E, Henderson D, Tamir O, et al. Health care-associated infections: a meta-analysis of costs and financial impact on the US health care system. JAMA Intern Med. 2013; 173:20392046. [PubMed: 23999949] 
14. Kwon JH, Olsen MA, Dubberke ER. The morbidity, mortality, and costs associated with Clostridium difficile infection. Infect Dis Clin North Am. 2015; 29:123-134. [PubMed: 25677706]

15. Kim KH, Fekety R, Batts DH, Brown D, Cudmore M, Silva J Jr, et al. Isolation of Clostridium difficile from the environment and contacts of patients with antibiotic-associated colitis. J Infect Dis. 1981; 143:42-50. [PubMed: 7217711]

16. Weber DJ, Anderson D, Rutala WA. The role of the surface environment in healthcare-associated infections. Curr Opin Infect Dis. 2013; 26:338-344. [PubMed: 23743816]

17. McFarland LV, Mulligan ME, Kwok RY, Stamm WE. Nosocomial acquisition of Clostridium difficile infection. N Engl J Med. 1989; 320:204-210. [PubMed: 2911306]

18. Vonberg RP, Kuijper EJ, Wilcox MH, Barbut F, Tull P, Gastmeier P, et al. Infection control measures to limit the spread of Clostridium difficile. Clin Microbiol Infect. 2008; 14(Suppl):2-20. [PubMed: 18412710]

19. Macgowan AP, Brown I, Feeney R, Lovering A, McCulloch SY, Reeves DS, et al. Clostridium difficile-associated diarrhoea and length of hospital stay. J Hosp Infect. 1995; 31:241-244. [PubMed: 8586796]

20. Barbut F, Petit JC. Epidemiology of Clostridium difficile-associated infections. Clin Microbiol Infect. 2001; 7:405-410. [PubMed: 11591202]

21. Abdelsattar ZM, Krapohl G, Alrahmani L, Banerjee M, Krell RW, Wong SL, et al. Postoperative burden of hospital-acquired Clostridium difficile infection. Infect Control Hosp Epidemiol. 2015; 36:40-46. [PubMed: 25627760]

22. Shaughnessy MK, Micielli RL, DePestel DD, Arndt J, Strachan CL, Welch KB, et al. Evaluation of hospital room assignment and acquisition of Clostridium difficile infection. Infect Control Hosp Epidemiol. 2011; 32:201-206. [PubMed: 21460503]

23. Lawrence SJ, Puzniak LA, Shadel BN, Gillespie KN, Kollef MH, Mundy LM. Clostridium difficile in the intensive care unit: epidemiology, costs, and colonization pressure. Infect Control Hosp Epidemiol. 2007; 28:123-130. [PubMed: 17265392]

24. Dubberke ER, Reske KA, Olsen MA, McMullen KM, Mayfield JL, McDonald LC, et al. Evaluation of Clostridium difficile-associated disease pressure as a risk factor for C difficileassociated disease. Arch Intern Med. 2007; 167:1092-1097. [PubMed: 17533213]

25. Eyre DW, Cule ML, Wilson DJ, Griffiths D, Vaughan A, O'Connor L, et al. Diverse sources of C. difficile infection identified on whole-genome sequencing. N Engl J Med. 2013; 369:1195-1205. [PubMed: 24066741]

26. Curtis DE, Hlady CS, Kanade G, Pemmaraju SV, Polgreen PM, Segre AM. Healthcare worker contact networks and the prevention of hospital-acquired infections. PLoS ONE. 2013; 8:e79906. [PubMed: 24386075]

27. Hornbeck T, Naylor D, Segre AM, Thomas G, Herman T, Polgreen PM. Using sensor networks to study the effect of peripatetic healthcare workers on the spread of hospital-associated infections. $\mathrm{J}$ Infect Dis. 2012; 206:1549-1557. [PubMed: 23045621]

28. Polgreen PM, Tassier TL, Pemmaraju SV, Segre AM. Prioritizing healthcare worker vaccinations on the basis of social network analysis. Infect Control Hosp Epidemiol. 2010; 31:893-900. [PubMed: 20649412]

29. Elixhauser A, Steiner C, Harris DR, Coffey RM. Comorbidity measures for use with administrative data. Med Care. 1998; 36:8-27. [PubMed: 9431328]

30. D’Agostino RB, Lee ML, Belanger AJ, Cupples LA, Anderson K, Kannel WB. Relation of pooled logistic regression to time dependent Cox regression analysis: the Framingham Heart Study. Stat Med. 1990; 9:1501-1515. [PubMed: 2281238]

31. Dubberke ER, Reske KA, Olsen MA, McMullen KM, Mayfield JL, McDonald LC, et al. Evaluation of Clostridium difficile-associated disease pressure as a risk factor for $\mathrm{C}$ difficileassociated disease. Arch Intern Med. 2007; 167:1092-1097. [PubMed: 17533213]

32. Gerding DN, Muto CA, Owens RC Jr. Measures to control and prevent Clostridium difficile infection. Clin Infect Dis. 2008; 46(Suppl):S43-S49. [PubMed: 18177221]

33. Hamel M, Zoutman D, O'Callaghan C. Exposure to hospital roommates as a risk factor for health care-associated infection. Am J Infect Control. 2010; 38:173-181. [PubMed: 20022405] 
34. Brown K, Valenta K, Fisman D, Simor A, Daneman N. Hospital ward antibiotic prescribing and the risks of Clostridium difficile infection. JAMA Intern Med. 2015; 175:626-633. [PubMed: 25705994]

35. Kuntz JL, Chrischilles EA, Pendergast JF, Herwaldt LA, Polgreen PM. Incidence of and risk factors for community-associated Clostridium difficile infection: a nested case-control study. BMC Infect Dis. 2011; 11:194. [PubMed: 21762504]

36. Wilcox MH, Mooney L, Bendall R, Settle CD, Fawley WN. A case-control study of communityassociated Clostridium difficile infection. J Antimicrob Chemother. 2008; 62:388-396. [PubMed: 18434341]

37. Kuntz JL, Polgreen PM. The importance of considering different healthcare settings when estimating the burden of clostridium difficile. Clin Infect Dis. 2015; 60:831-836. [PubMed: 25477426] 


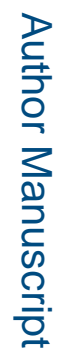

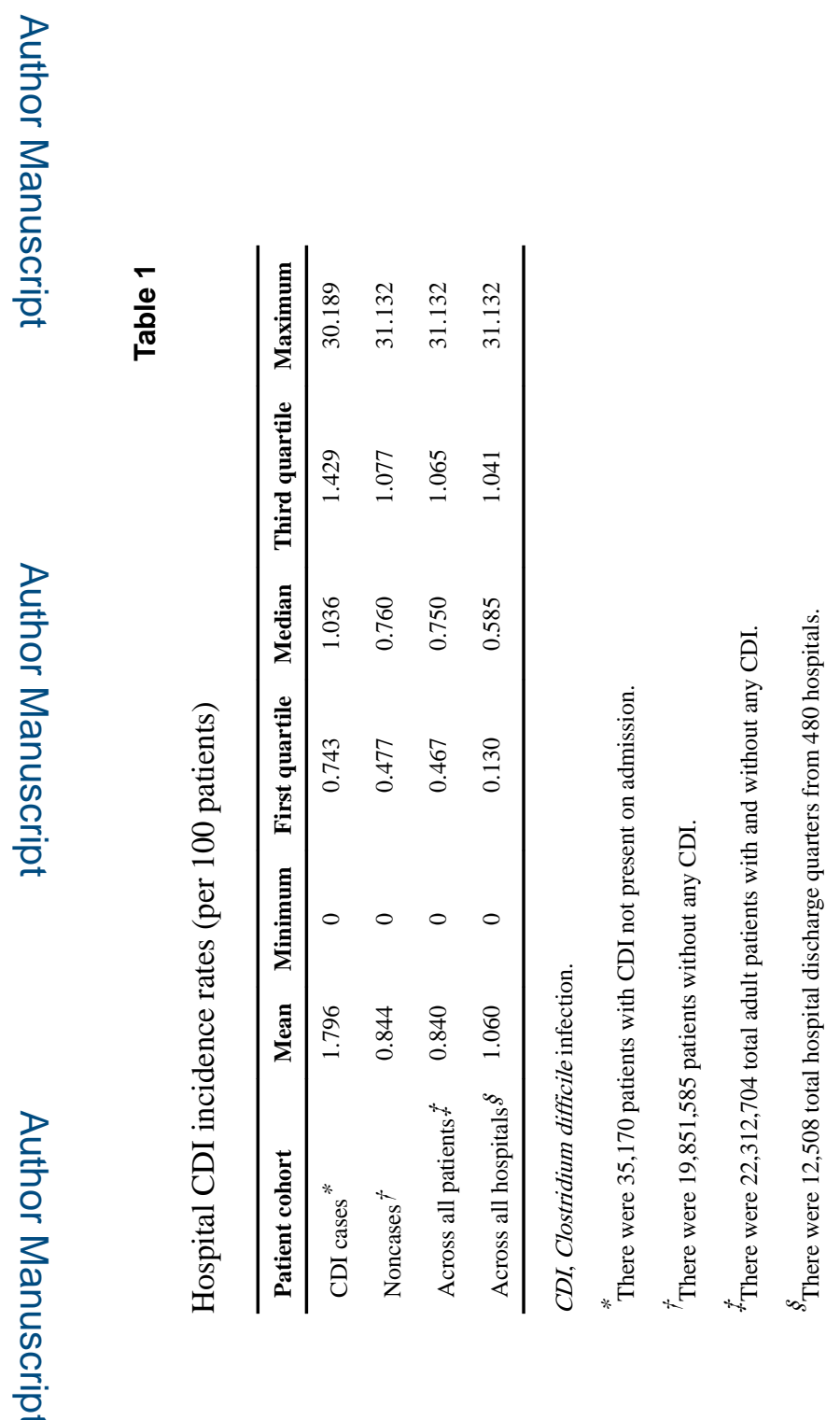

Am J Infect Control. Author manuscript; available in PMC 2017 July 01. 


\section{Table 2}

\section{Results of the multivariable analysis}

\begin{tabular}{|c|c|}
\hline Variable & Odds Ratio (95\% CI) \\
\hline CDI incidence & $1.183(1.173-1.193)$ \\
\hline \multicolumn{2}{|l|}{ Hospital size, quintile (discharges per quarter) } \\
\hline$<299$ & Reference \\
\hline 299-972 & $1.651(1.467-1.858)$ \\
\hline $973-2,121$ & $1.989(1.750-2.262)$ \\
\hline $2,122-4,116$ & $2.280(2.007-2.590)$ \\
\hline$>4,116$ & $2.589(2.277-2.942)$ \\
\hline Hospital average patient age (y) & $1.020(1.016-1.024)$ \\
\hline Hospital percent older adults (>65 y) & $0.985(0.982-0.988)$ \\
\hline Hospital percent Medicaid & $0.998(0.997-0.999)$ \\
\hline Previous admission $560 \mathrm{~d}$ & $1.635(1.590-1.680)$ \\
\hline Length of stay & $1.003(1.003-1.004)$ \\
\hline Age & $1.010(1.009-1.011)$ \\
\hline Female & $1.052(1.025-1.079)$ \\
\hline Nonwhite & $0.950(0.924-0.977)$ \\
\hline No. of procedures & $1.116(1.112-1.120)$ \\
\hline No. of diagnoses & $1.130(1.127-1.134)$ \\
\hline \multicolumn{2}{|l|}{ Admission source } \\
\hline Routine or other & Reference \\
\hline Another hospital & $1.171(1.115-1.229)$ \\
\hline Skilled nursing or intermediate care & $1.442(1.317-1.578)$ \\
\hline Other health facility & $0.990(0.892-1.098)$ \\
\hline Emergency department & $0.998(0.963-1.034)$ \\
\hline Intra-abdominal or gastrointestinal procedure & $1.318(1.274-1.363)$ \\
\hline Mechanical ventilation & $1.569(1.516-1.624)$ \\
\hline Hemodialysis & $1.163(1.116-1.212)$ \\
\hline Peritoneal dialysis & $1.320(1.089-1.599)$ \\
\hline Cancer chemotherapy & $3.308(3.105-3.523)$ \\
\hline Enteral and parenteral nutrition & $1.516(1.454-1.580)$ \\
\hline \multicolumn{2}{|l|}{ Elixhauser comorbidities } \\
\hline Alcohol abuse & $0.931(0.877-0.988)$ \\
\hline Deficiency anemias & $1.071(1.043-1.100)$ \\
\hline Cardiac arrhythmias & $0.931(0.904-0.958)$ \\
\hline Congestive heart failure & $1.126(1.091-1.162)$ \\
\hline Chronic pulmonary disease & $0.929(0.902-0.956)$ \\
\hline Coagulopathy & $1.068(1.030-1.108)$ \\
\hline Diabetes (uncomplicated) & $0.910(0.881-0.939)$ \\
\hline Diabetes with chronic complications & $0.797(0.764-0.832)$ \\
\hline Drug abuse & $0.739(0.685-0.797)$ \\
\hline
\end{tabular}




\begin{tabular}{lc}
\hline Variable & Odds Ratio $(\mathbf{9 5 \%} \mathbf{C I})$ \\
\hline Hypertension & $0.893(0.868-0.918)$ \\
Hypothyroidism & $0.896(0.864-0.929)$ \\
Liver disease & $0.942(0.893-0.993)$ \\
Fluid and electrolyte disorders & $1.591(1.547-1.637)$ \\
Metastatic cancer & $0.889(0.840-0.942)$ \\
Other neurologic disorders & $1.051(1.013-1.090)$ \\
Obesity & $0.848(0.813-0.884)$ \\
Paralysis & $1.211(1.158-1.266)$ \\
Peripheral vascular disorders & $0.813(0.781-0.846)$ \\
Pulmonary circulation disorders & $1.108(1.049-1.170)$ \\
Valvular disease & $0.809(0.770-0.851)$ \\
Weight loss & $1.470(1.424-1.518)$ \\
\hline
\end{tabular}

CI, confidence interval; CDI, Clostridium difficile infection. 